Marquette University

e-Publications@Marquette

Mathematics, Statistics and Computer Science

Mathematics, Statistics and Computer Science,

Faculty Research and Publications

Department of

$10-1-2012$

\title{
On Lattices of Varieties of Restriction Semigroups
}

Peter R. Jones

Marquette University, peter.jones@marquette.edu

Accepted version. Semigroup Forum, Vol. 86, No. 2 (April 2013; 337-361) . DOI. (C) 2013 Springer. Used with permission.

Shareable Link. Provided by the Springer Nature SharedIt content-sharing initiative. 


\title{
On lattices of varieties of restriction semigroups
}

\author{
Peter R. Jones
}

September 19, 2012

\begin{abstract}
The left restriction semigroups have arisen in a number of contexts, one being as the abstract characterization of semigroups of partial maps, another as the 'weakly left $E$ ample' semigroups of the 'York school', and, more recently as a variety of unary semigroups defined by a set of simple identities. We initiate a study of the lattice of varieties of such semigroups and, in parallel, of their two-sided versions, the restriction semigroups. Although at the very bottom of the respective lattices the behaviour is akin to that of varieties of inverse semigroups, more interesting features are soon found in the minimal varieties that do not consist of semilattices of monoids, associated with certain 'forbidden' semigroups. There are two such in the one-sided case, three in the two-sided case. Also of interest in the one-sided case are the varieties consisting of unions of monoids, far indeed from any analogue for inverse semigroups. In a sequel, the author will show, in the two-sided case, that some rather surprising behavior is observed at the next 'level' of the lattice of varieties.
\end{abstract}

The restriction semigroups, in both the one- and the two-sided incarnations, have risen to prominence at the confluence of several historical strands of research. The left restriction semigroups, in particular, abstractly characterize semigroups of partial maps of a set; both versions also arise at the ends of sequences of natural generalizations - if perhaps only in hindsight - of the ample semigroups introduced, in other terminology, by Fountain $[7,8]$. (The historical connection is witnessed by the alternative names 'weakly [left-] $E$-ample semigroups'.) The primary virtue of this more recent viewpoint is that the classes under consideration form varieties under natural signatures. The study of the lattices of varieties, in the respective contexts, provides a systematic way to study known classes, and to discover new and interesting classes, of such semigroups. Since our presentation will be largely self-contained, based on the identities that define the classes under study, we have deferred to an appendix more explicit discussion of the connection between this approach and the various historical strands alluded to above.

Ultimately the variety of restriction semigroups is, in fact, that generated by inverse semigroups, when the inverse operation in the latter is 'forgotten' and they are considered as 'biunary' semigroups $\left(S, \cdot{ }^{+},{ }^{*}\right)$, where $x^{+}=x x^{-1}$ and $x^{*}=x^{-1} x$; the left restriction semigroups are the unary semigroups $\left(S, \cdot,^{+}\right)$that result from retaining only the former operation. Lying between inverse semigroups and 'plain' semigroups, similarities with the well-studied lattice of varieties of inverse semigroups decrease as more operations are forgotten, as might be expected. 
We begin this investigation at the 'bottom' of the respective lattices, by considering the role of the variety $\mathbf{M}$ of monoids (which play here the role that groups play for inverse semigroups) and then the lattice of subvarieties of the join of $\mathbf{M}$ with the variety $\mathbf{S L}$ of semilattices, which are considered as restriction semigroups, appropriate to the context. In either context, this join consists of the semilattices of monoids. This material consists of fairly straightforward extensions of the theory of inverse semigroup varieties.

Of more interest - and diverging from inverse semigroup theory - are the varieties minimal with respect to not lying within this join, each corresponding to a 'forbidden' semigroup. In the case of restriction semigroups, there are three such semigroups, namely the 'upper and lower triangles' $B^{+}$and $B^{-}$, respectively, of the bicyclic semigroup, and the subsemigroup $B_{0}$ of the five-element Brandt semigroup $B_{2}$ that is obtained by omitting one of the two nonidempotents. The relationships among the varieties they generate are also considered.

In the case of left restriction semigroups, a further layer appears: the varieties that consist of unions of monoids (but not semilattices of monoids). These semigroups seem to have received little, if any, specific attention heretofore, and warrant further study. In the one-sided situation, there are instead two three-element 'forbidden' semigroups, $L_{2}^{1}$ and $D$, relative to the join of $\mathbf{M}$ and SL. One discriminates varieties of unions of monoids from those of semilattices of monoids; the second discriminates the former from varieties in general.

While a few of our results no doubt simply place folklore in a varietal context, we hope to stimulate further study of this topic. In a sequel [17], the author will consider varieties generated by (the reducts of) Brandt semigroups, and in particular by the semigroups $B_{2}$ and $B_{0}$. The situation is remarkably more complex than for inverse semigroup varieties.

As mentioned below, Cornock in her thesis [3] has investigated varieties of [left] restriction semigroups from a rather different perspective.

\section{Background}

For the purposes of this work, it is appropriate to define [left] restriction semigroups by means of their identities. A left restriction semigroup is a unary $\operatorname{semigroup~}\left(S, \cdot{ }^{+}\right)$that satisfies

$$
x^{+} x=x ; \quad\left(x^{+} y\right)^{+}=x^{+} y^{+} ; \quad x^{+} y^{+}=y^{+} x^{+} ; \quad x y^{+}=(x y)^{+} x .
$$

(We take this particular definition from [12], where it is attributed to Jackson and Stokes [15].) The last identity (or a variation of it) is often termed the 'left ample' condition.

From the first two identities it follows that for all $x \in S, x^{+}$is idempotent and then, in conjunction with the second identity, that $\left(x^{+}\right)^{+}=x^{+}$. We term these idempotents the projections of $S$. Denote the set of projections by $P_{S}$ and the set of all idempotents by $E_{S}$. Although, by the third identity, $P_{S}$ is a semilattice, this need by no means be true of $E_{S}$. In the usual way, $E_{S}$ is partially ordered by $e \leq f$ if $e=e f=f e$. (Note that, traditionally, the projections have often been termed 'distinguished idempotents' and the semilattice of distinguished idempotents has been denoted $E$ : see the Appendix for further discussion.) 
The following consequences of the identities are well known. We include proofs only to illustrate the usage of the identities.

LEMMA 1.1 A left restriction semigroup satisfies $x^{+} \geq(x y)^{+}$and $(x y)^{+}=\left(x y^{+}\right)^{+}$.

Proof. Let $S$ be such a semigroup and $x, y \in S$. Applying the first defining identity, then the second and the fact that $\left(x^{+}\right)^{+}=x^{+}$, noted above, $(x y)^{+}=\left(x^{+}(x y)\right)^{+}=x^{+}(x y)^{+}$, as required. Next, applying the left ample identity, then the second identity and the inequality just obtained, $\left(x y^{+}\right)^{+}=\left((x y)^{+} x\right)^{+}=(x y)^{+} x^{+}=(x y)^{+}$.

A right restriction semigroup is a unary semigroup $\left(S, \cdot,{ }^{*}\right)$ that satisfies the 'dual' identities, obtained by replacing ${ }^{+}$by ${ }^{*}$ and reversing the order of each expression. We shall make little explicit mention of such semigroups, since duality may be invoked to yield the analogues of results about left restriction semigroups.

A restriction semigroup is a biunary semigroup $\left(S, \cdot,{ }^{+},{ }^{*}\right)$ that is a left restriction semigroup with respect to ${ }^{+}$, a right restriction semigroup with respect to ${ }^{*}$, and satisfies $\left(x^{+}\right)^{*}=x^{+}$and $\left(x^{*}\right)^{+}=x^{*}$.

The term 'restriction' is relatively recent, deriving from its use by Cockett and Lack [2] in one of the several sources of these semigroups (and of categories, in their paper). Until quite recently, the term 'weakly $E$-ample' was used, providing evidence of a succession of generalizations - by the so-called York school - of Fountain's 'ample semigroups' (though different terminology again was used in the original papers $[7,8]$ ). The definitions and fundamental properties provided below - and several of the structural theorems that underpin the lattice-theoretical results found herein - have precursors that we will not cite. In fact, when expressed in the language of varieties and identities, many definitions (for example the generalized Green's relations) have very simple formulations that require no knowledge of their historical development.

Nevertheless, in an appendix we provide a brief summary of the interpretation of this background in the language of the York school. Much fuller exposition of this material may be found in the work of Gould [11] and Hollings [13], for instance, and in the thesis of Cornock [3]. This last citation in fact contains extensive work on varieties of restriction semigroups, in both oneand two-sided guises, focusing on the existence and construction of appropriate proper covers. We view the current paper as complementary to her work. However, it will receive frequent mention.

In the context of this work, an inverse semigroup $\left(S, \cdot{ }^{-1}\right)$ may be regarded as a restriction semigroup by setting $x^{+}=x x^{-1}$ and $x^{*}=x^{-1} x$ and 'forgetting' the inverse operation. In that case, $P_{S}=E_{S}$. It may be regarded as a left [right] restriction semigroup by admitting only the former [latter] operation. This source of examples may be expanded upon by noting that any subsemigroup that is full (contains all its idempotents) again induces such a [left, right] restriction subsemigroup. Each such semigroup is, in fact, [left, right] ample (see the appendix).

For the purposes of this paper, the relevant generalized Green's relations may be defined as follows. In a left restriction semigroup, $\mathbb{R}=\left\{(a, b): a^{+}=b^{+}\right\}$. The second statement in Lemma 1.1 simply asserts that $\mathbb{R}$ is a left congruence. In a right restriction semigroup, $\mathbb{L}=\left\{(a, b): a^{*}=b^{*}\right\}$. In a restriction semigroup, $\mathbb{H}=\mathbb{L} \cap \mathbb{R}$. It follows easily from Lemma 1.1 
and its dual that each contains the corresponding usual Green's relation. In the case of a [left] restriction semigroup that is induced from a (full subsemigroup of an) inverse semigroup, as above, each clearly coincides with the restriction of the usual Green's relation. Refer to the Appendix for the rationale behind this streamlining of the traditional notation $\widetilde{\mathcal{R}}_{E}, \widetilde{\mathcal{L}}_{E}$ and $\widetilde{\mathcal{H}}_{E}$.

In general, the terms 'homomorphism' and 'congruence' will be used appropriate to context; that is, they should respect the unary operation for left restriction semigroups and both unary operations for restriction semigroups.

In the standard terminology, restriction semigroups $S$ with $\left|P_{S}\right|=1$ are termed reduced. Since, in essence, they are just monoids, regarded as restriction semigroups by setting $a^{+}=a^{*}=$ 1 for all $a$, we will generally omit the qualifier 'reduced', except in case of possible ambiguity. The least monoid congruence on a [left] restriction semigroup $S$ is denoted $\sigma$ and is just the least semigroup congruence that identifies all of its projections (as noted in [11]). Then, whether in a left restriction or a restriction semigroup, $a \sigma b$ if and only if $e a=e b$ for some $e \in P_{S}$. If $S$ is an inverse semigroup, $\sigma$ is the least group congruence on $S$. (Once more, a fuller exposition may be found in [3]).

A left restriction semigroup is proper if $\sigma \cap \mathbb{R}=\iota$, where $\iota$ denotes the identical relation. A restriction semigroup is proper if both this equation and its dual hold. A proper cover for a [left] restriction semigroup $S$ consists of a proper [left] restriction semigroup $T$ and a $P$-separating homomorphism from $T$ onto $S$. The existence of proper covers for restriction semigroups in general (there being many precursors) was demonstrated in [10, Theorem 7.1]; a one-sided version was given by Cornock [3, Theorem 1.9.6]. The author provided an elementary construction that covers both cases in [16]. The details are not needed in the sequel.

RESULT 1.2 Every [left] restriction semigroup has a proper cover.

On any type of restriction semigroup $S, \mu$ denotes the greatest congruence, of the appropriate type, that is $P$-separating, that is, separates $P_{S}$, and $S$ is called fundamental if $\mu=\iota$. In particular, $S / \mu$ is fundamental. Although sometimes the one-sided versions of these definitions have received names reflecting their origins, the meanings of the terms will be clear from context in this paper. If $S$ is a left restriction semigroup, then $\mu$ is the largest (unary) congruence that is contained in $\mathbb{R}$. It is well known that if $a, b \in S$, then $a \mu b$ if and only if $(a e)^{+}=(b e)^{+}$for all $e \in P_{S}^{1}$. In the case of a restriction semigroup, $\mu$ is the greatest (biunary) congruence on $S$ that is contained in $\mathbb{H}$. In this case, $a \mu b$ if and only if $(a e)^{+}=(b e)^{+}$for all $e \in P_{S}$, and if and only if $(e a)^{*}=(e b)^{*}$ for all $e \in P_{S}$.

LEMMA 1.3 If a [left] restriction semigroup $S$ is proper, then $\sigma \cap \mu=\iota$, so $S$ is a subdirect product of the monoid $S / \sigma$ and the fundamental [left] restriction semigroup $S / \mu$.

Proof. This is immediate from the definition of properness, since $\mu \subseteq \mathbb{R}$ in either situation. $\square$

$\mathrm{A}^{+}{ }_{-}$ideal $I$ of a left restriction semigroup $S$ is an ideal of $S$ that is also a left restriction subsemigroup. It is easily seen that the Rees quotient semigroup $S / I$ is again a left restriction 
semigroup. We will term the obvious two-sided analogue a restriction-ideal. (As usual, for technical reasons it is convenient to allow the empty set to be an ideal and, in that case, to put $S / I=S$.)

\subsection{Varieties and free objects}

We refer the reader to standard texts such as [1] for universal algebraic background, of which only the most basic is needed. We may treat the one-sided and two-sided cases in parallel.

Denote by $\mathbf{L R}$ the variety of left restriction semigroups and by $\mathbf{R}$ the variety of restriction semigroups, in the respective signatures.

In either context, T, $\mathbf{M}$ and $\mathbf{S L}$ will denote the varieties of trivial semigroups, monoids, and semilattices, respectively. Either as a subvariety of $\mathbf{L R}$ or a subvariety of $\mathbf{R}, \mathbf{M}$ may be defined by the identity $x^{+}=y^{+}$. Note that, in either context, subvarieties of $\mathbf{M}$ are essentially varieties of monoids, and we shall treat them as such. For instance, $\mathbf{M C}$ refers to the variety of commutative monoids. The variety SL may be defined by the identity $x=x^{+}$in either context. Care must be taken to distinguish this variety from the variety MSL of semilattice monoids, defined by $x^{+}=y^{+}, x^{2}=x, x y=y x$. Note that a variety $\mathbf{V}$ of [left] restriction semigroups consists of monoids if and only if $\mathbf{V} \cap \mathbf{S L}=\mathbf{T}$.

Other varieties will be introduced as needed. If $S$ is a [left] restriction semigroup, then $\mathbf{V}(S)$ will denote the variety of [left] restriction semigroups that it generates. If $\mathbf{V}$ is a variety of [left] restriction semigroups, then $\mathcal{L}(\mathbf{V})$ denotes its lattice of subvarieties.

The inverse semigroups, when regarded as [left] restriction semigroups, do not form a variety in either context, since they are not closed under taking [left] restriction subsemigroups. However, they play an important role, since $\mathbf{L R}$ and $\mathbf{R}$ are each generated by the (reducts of) inverse semigroups. This follows from the descriptions of the free [left] restriction semigroups $[10,9]$ and also from quite elementary arguments, as demonstrated by the author in [16]. Either from the cited descriptions, or from the latter facts, the following can be obtained.

RESULT 1.4 Let $X$ be a nonempty set. The free restriction semigroup $F \mathbf{R}_{X}$ on $X$ is isomorphic to the subsemigroup of the free inverse semigroup $F I_{X}$ on $X$ that is generated, as a restriction semigroup, by $X$, and comprises the complete inverse image of the free monoid on $X$ under the homomorphism $F I_{X} \longrightarrow F G_{X}$ onto the free group on $X$.

The free left restriction semigroup $F \mathbf{L R}_{X}$ is isomorphic to the subsemigroup of $F I_{X}$ that is generated, as a left restriction semigroup, by $X$.

We only need the details of these representations in the monogenic case, that is, when $X=\{x\}$. We use the representation inherent in the Munn representation of free inverse semigroups [21]: its elements are uniquely representable in the form $\left(x^{-m} x^{m}\right) x^{k}\left(x^{n} x^{-n}\right)$, where $m, n, m+k, n+k, m+k+n \geq 0$ and not all of $m, k, n$ are zero. (Here $x^{0}$ represents an adjoined identity.) Clearly such an element is mapped to $x^{k}$ in the free group image. (The equivalent description in [22, Proposition IX.1.9] instead uses the triple $(m, k, n)$ to represent the product above.) A useful fact, true for free inverse semigroups in general, is that $F I_{x}$ is combinatorial, that is, $\mathcal{H}=\iota$. 
RESULT 1.5 In terms of the above representation of $F I_{x}$ and the operations in restriction semigroups, the elements of $F \mathbf{R}_{x}$ may be uniquely expressed in the form $\left(x^{m}\right)^{*} x^{k}\left(x^{n}\right)^{+}$, $m, n, k \geq 0, m+k+n \geq 1$. Under that representation, the relations $\mathbb{R}$ and $\mathbb{L}$ are just the restrictions of the usual Green's relations $\mathcal{R}$ and $\mathcal{L}$, and so $\mathbb{H}=\iota$. The projections of $F \mathbf{R}_{x}$ are just its idempotents: the elements $\left(x^{m}\right)^{*}\left(x^{n}\right)^{+}, m, n \geq 0, m+n \geq 1$.

RESULT 1.6 In terms of the above representation of $F I_{x}$ and the operations in left restriction semigroups, the elements of $F \mathbf{L R}_{x}$ may be uniquely expressed in the form $x^{k}\left(x^{n}\right)^{+}, n, k \geq 0$, $k+n \geq 1$. Under that representation, the relation $\mathbb{R}$ is the restriction of the usual Green's relation $\mathcal{R}$.

The key equation for computation in monogenic left restriction semigroups is $x^{k}\left(x^{n}\right)^{+}=$ $\left(x^{k+n}\right)^{+} x^{k}$, based on the left ample identity. In particular, $\left(x^{k}\left(x^{n}\right)^{+}\right)^{+}=\left(x^{k+n}\right)^{+}$. This provides a slightly more convenient representation of its elements: in the form $\left(x^{p}\right)^{+} x^{q}, p \geq 1$, $0 \leq q \leq p$. For a given value of $p$, the corresponding elements then comprise the $\mathbb{R}$-class of $x^{p}$ in $F \mathbf{L} \mathbf{R}_{x}$, since $\left(\left(x^{p}\right)^{+} x^{q}\right)^{+}=\left(x^{p}\right)^{+}\left(x^{q}\right)^{+}=\left(x^{p}\right)^{+}$.

Two particular monogenic inverse semigroups play important roles in the general theory and also in the study of varieties of inverse semigroups. Not surprisingly, their reducts - more often the reducts of certain subsemigroups and quotients - will play important roles in this paper.

Recall that the bicyclic monoid is presented, as a 'plain' monoid, by $B=\langle a, b \mid a b=1\rangle$ or, as a semigroup, by $\left\langle a, b \mid a=a b a=a^{2} b, b=b a b=a b^{2}\right\rangle$. As is well known, its elements are uniquely representable in the form $b^{m} a^{n}$, for nonnegative integers $m, n$ (where $a^{0}=b^{0}=1$ ). It is an inverse monoid, where $\left(b^{m} a^{n}\right)^{-1}=b^{n} a^{m}$, and is presented as such by $\left\langle a \mid a a^{-1} \geq a^{-1} a\right\rangle$. If we put $e_{m}=b^{m} a^{m}$, for $m \geq 0$, then $E_{B}$ is the semilattice $1=e_{0}>e_{1}>e_{2}>\cdots$.

Denote by $B_{2}$ the five-element combinatorial Brandt inverse semigroup, with semigroup presentation $\left\langle a, b \mid a b a=a, b a b=b, a^{2}=b^{2}=0\right\rangle=\{a, b, a b, b a, 0\}$. Regarded as an inverse semigroup, $B_{2}=\left\{a, a^{-1}, a a^{-1}, a^{-1} a, 0\right\}$.

\section{$1.2 \quad$ Varieties of inverse semigroups}

For reference in the sequel, we summarize the relevant facts. Denote by $\mathbf{I}$ the variety of inverse semigroups, in the signature $\left(\cdot,,^{-1}\right)$, and by $\mathcal{L}(\mathbf{I})$ its lattice of subvarieties. Denote by $\mathbf{G}$ the subvariety of groups and (once again) by $\mathbf{S L}$ the subvariety of semilattices.

RESULT 1.7 (1) [22, Theorems XII.2.8, XII.3.2] On $\mathcal{L}(\mathbf{I})$, the map $\mathbf{V} \longrightarrow \mathbf{V} \vee \mathbf{G}$ is a complete lattice homomorphism and the map $\mathbf{V} \longrightarrow \mathbf{V} \cap \mathbf{G}$ is a lattice homomorphism;

(2) [22, cf Corollary XII.4.5] The join $\mathbf{G} \vee \mathbf{S L}$ consists of the semilattices of groups (Clifford semigroups), and is defined by $x x^{-1}=x^{-1} x$;

(3) [22, from Theorem XII.4.16] The sublattice $\mathcal{L}(\mathbf{G} \vee \mathbf{S L})$ of $\mathcal{L}(\mathbf{I})$ is isomorphic to the direct product of the two-element lattice $\mathcal{L}(\mathbf{S L})$ and the lattice $\mathcal{L}(\mathbf{G})$, under the map $\mathbf{V} \mapsto(\mathbf{V} \cap \mathbf{S L}) \vee(\mathbf{V} \cap \mathbf{G}) ;$ 
(4) [22, Proposition XII.4.13(ii)] The variety generated by $B_{2}$ is the smallest variety that does not consist of semilattices of groups.

\section{Varieties of restriction semigroups}

\subsection{Joins and meets with $\mathrm{M}$}

The behaviour of the variety $\mathbf{M}$ in the lattice of varieties of restriction semigroups parallels that of the variety of groups in the lattice of varieties of inverse semigroups, cited in Result 1.7 above.

THEOREM 2.1 If $\mathbf{V} \in \mathcal{L}(\mathbf{R})$, then $\mathbf{V} \vee \mathbf{M}=\{S \in \mathbf{R}: S / \mu \in \mathbf{V}\}$. Hence the map $\mathbf{V} \longrightarrow$ $\mathbf{V} \vee \mathbf{M}$ is a complete lattice homomorphism.

If $\mathbf{V}$ is defined by the identities $u_{i}=v_{i}, i \in I$, then $\mathbf{V} \vee \mathbf{M}$ is defined by the identities $\left(u_{i} x\right)^{+}=\left(v_{i} x\right)^{+}, i \in I$, where $x$ is a letter distinct from any in the original set of identities.

Proof. To prove the first assertion, suppose $S / \mu \in \mathbf{V}$. Let $T$ be a proper cover of $S$, according to Result 1.2. Then, by Lemma $1.3, S$ is a subdirect product of $T / \mu$ and $T / \sigma \in \mathbf{M}$. But the covering map $T \longrightarrow S$ is $P$-separating and so $T / \mu \cong S / \mu \in \mathbf{V}$. Hence $T$, and therefore $S$, belongs to $\mathbf{V} \vee \mathbf{M}$.

Conversely, any member $S$ of $\mathbf{V} \vee \mathbf{M}$ is a homomorphic image of a (biunary) subsemigroup $W$, say, of $U=T \times M$, where $T \in \mathbf{V}$ and $M \in \mathbf{M}$. Now the relation $\mathbb{H}$ on $U$ is clearly the product of the corresponding relations on $T$ and $M$. Thus the same is true of $\mu$, so that $U / \mu \cong T / \mu \times M / \mu$. But $M / \mu$ is trivial, so $U / \mu \cong T / \mu \in \mathbf{V}$. Next, observe that the restriction $\eta$, say, of $\mu$ to $W$ is $P$-separating, $\eta \subseteq \mu_{W}$ (the $\mu$-relation on $W$ ) and so there is a homomorphism from $W / \eta$ onto $W / \mu_{W}$. But $W / \eta \leq U / \mu$. Hence $W / \mu_{W} \in \mathbf{V}$. Finally, if the surjective homomorphism $W \longrightarrow S$ induces the congruence $\rho$, say, on $W$, then the congruence $\left(\rho \vee \mu_{W}\right) / \rho$ on $S$ is $P$-separating and therefore maps onto $S / \mu$. Thus if $W / \mu_{W} \in \mathbf{V}$ then $S / \mu_{S} \in \mathbf{V}$. This completes the proof of the opposite inclusion.

The second assertion follows immediately.

The final statement follows from the description of the congruence $\mu$ in Section 1: $a \mu b$ if and only if $(a e)^{+}=(b e)^{+}$for all $e \in P_{S}$, noting that, by Lemma 1.1, the second equation is equivalent to $(a x)^{+}=(b x)^{+}$for all $x \in S$.

That the map $\mathbf{V} \longrightarrow \mathbf{V} \cap \mathbf{M}$ is a homomorphism is best proved through consideration of fully invariant congruences on free restriction semigroups. That is, it needs to be shown that on the free restriction semigroup of infinite rank, the equation $(\alpha \vee \sigma) \cap(\beta \vee \sigma)=(\alpha \cap \beta) \vee \sigma$ holds for any fully invariant congruences $\alpha$ and $\beta$. In fact it holds in general, as a consequence of the next lemma, which generalizes the well known result [22, Lemma III.5.4] for inverse semigroups.

LEMMA 2.2 If $\rho$ is any congruence on a restriction semigroup $S$, then $\rho \vee \sigma=\{(a, b):$ ea $\rho$ eb for some $\left.e \in P_{S}\right\}$. Hence for any congruences $\rho$ and $\tau,(\rho \vee \sigma) \cap(\tau \vee \sigma)=(\rho \cap \tau) \vee \sigma$. 
Proof. Recall that $\sigma=\left\{(a, b): e a=e b\right.$ for some $\left.e \in P_{S}\right\}$. Thus if ea $\rho e b$ for some $e \in P_{S}$, then $a \sigma e a \rho e b \sigma b$. Conversely, suppose $(a, b) \in \rho \vee \sigma$. Thus $a \rho x_{1} \sigma y_{1} \rho x_{2} \cdots \sigma y_{n}=b$, for suitable elements of $S$. For $i=1,2, \ldots, n$, there exist $e_{i} \in P_{S}$ such that $e_{i} x_{i}=e_{i} y_{i}$. Putting $e=e_{1} \cdots e_{n}$, it follows by commutativity of $P_{S}$ that $e \in P_{S}$ and $e a \rho e b$, as required.

Now if $(a, b) \in(\rho \vee \sigma) \cap(\tau \vee \sigma)$, there exist $e, f \in P_{S}$ such that $e a \rho e b$ and $f a \tau f b$. Then $e f=f e \in P_{S}$ and efa $\rho \cap \tau$ efb.

In view of the remarks above, the next result is now immediate.

THEOREM 2.3 The map $\mathbf{V} \longrightarrow \mathbf{V} \cap \mathbf{M}, \mathbf{V} \in \mathcal{L}(\mathbf{R})$ is a homomorphism.

We use the example given by Reilly (see [22, Example XII.3.6]), which demonstrated that the variety of groups does not separate the lattice of varieties of inverse semigroups, to show that $\mathbf{M}$ does not separate the lattice $\mathcal{L}(\mathbf{R})$.

EXAMPLE 2.4 Let $C=\langle a\rangle$ be an infinite cyclic group and put $G=C \times C$. Let $S$ be the Brandt semigroup $\mathcal{M}^{0}(I, G, I, \Delta)$ over $G$, where $I=\{1,2\}$. Let $\alpha_{1}, \alpha_{2}$ denote, respectively, the identity map on $G$ and the automorphism of $G$ that sends $(x, y)$ to $(y, x)$. Let $T=S \cup G$, where the multiplication extends that on $S$ and $G$, and for $g \in G$ and $(i, a, j) \in S$,

$$
g(i, a, j)=\left(i,\left(g \alpha_{i}\right) a, j\right) \quad \text { and } \quad(i, a, j) g=\left(i, a\left(g \alpha_{j}\right), j\right) .
$$

Then $T$ is an inverse semigroup, $\mathcal{H}$ is a congruence and is thus $\mu$, and $T / \mu \cong B_{2}^{1}$. Regarding $T$ and $B_{2}^{1}$ as restriction semigroups, it follows from Theorem 2.1 that $\mathbf{V}(T) \subseteq \mathbf{V}\left(B_{2}^{1}\right) \vee \mathbf{M}$. We have to replace the identities used in the case of inverse semigroup varieties by biunary ones. In fact, only the unary operation ${ }^{+}$is needed, which will be of relevance in the sequel. We first show the identity $x x^{+} y y^{+}=y y^{+} x x^{+}$is satisfied in $T$. If $x, y \in G$ this is clear; if $x$ is a nonidempotent of $S$, then $x x^{+}=0$, and similarly for $y$; if both $x$ and $y$ are idempotents (and thus projections), satisfaction is also clear. The remaining case is, without loss of generality, when $x \in G$ and $y=e$ is a nonzero idempotent of $S$, in which case $x x^{+} y y^{+}=y y^{+} x x^{+}$reduces to $x e=e x$. But since $\mathcal{H}$ is a congruence, $x e \mathcal{H} e$ and so $x e=e x e=e x$. As a consequence, any monoid in $\mathbf{V}(T)$ is commutative, that is, $\mathbf{V}(T) \cap \mathbf{M} \subseteq \mathbf{M C}$. But $C$ generates MC, so equality holds.

Now put $\mathbf{U}=\mathbf{V}\left(B_{2}^{1}\right) \vee \mathbf{M C}$. From the previous paragraph, $\mathbf{V}(T) \vee \mathbf{M}=\mathbf{U} \vee \mathbf{M}$ and $\mathbf{V}(T) \cap \mathbf{M}=\mathbf{M C}=\mathbf{U} \cap \mathbf{M}$.

The identity $(x y)^{+} y x=(y x)^{+} x y$ that we now apply will also appear in Proposition 2.11. It is routinely checked that it is satisfied in $B_{2}^{1}$ and thus in $\mathbf{U}$. We now show that it is not satisfied in $T$, so that $\mathbf{U} \neq \mathbf{V}(T)$ : if $x=(2,(a, 1), 1) \in S$ and $y=(a, 1) \in G$, then $x y=\left(2,\left(a^{2}, 1\right), 1\right)$ and $y x=(2,(a, a), 1)$; but $x y \mathcal{H} y x$, so $(x y)^{+}(y x)=y x$ and $(y x)^{+} x y=x y$, a contradiction. Thus we have proved the following.

PROPOSITION 2.5 The variety of monoids does not separate the lattice $\mathbf{R}$. 
It should be noted that we chose to use Reilly's example because it is well known. The argument used above remains valid if the infinite cyclic group is replaced by the infinite cyclic monoid, for instance; likewise, $S$ may be replaced by certain of its full subsemigroups.

Looking ahead to the next subsection, if $\mathbf{V}, \mathbf{W} \in \mathcal{L}(\mathbf{S L} \vee \mathbf{M})$ and $\mathbf{V} \vee \mathbf{M}=\mathbf{W} \vee \mathbf{M}$, it follows from Theorem 2.8 that $\mathbf{V} \cap \mathbf{S L}=\mathbf{W} \cap \mathbf{S L}$. Thus $\mathbf{M}$ separates $\mathcal{L}(\mathbf{S L} \vee \mathbf{M})$.

\subsection{Varieties of restriction semigroups consisting of semilattices of monoids}

In the context of restriction semigroups, the term $S$ is a semilattice $Y$ of monoids $S_{\alpha}$ specifically requires that $Y \in \mathbf{S L}$, that the map $S \longrightarrow Y$ be a biunary homomorphism, and that each $S_{\alpha}$ belong to $\mathbf{M}$. The definition of strong semilattice of monoids requires that the structure homomorphisms $S_{\alpha} \longrightarrow S_{\beta}, \alpha \geq \beta$, be monoidal (that is, respect the identity elements).

A (biunary) subsemigroup $T$ of a restriction semigroup $S$ is a submonoid if it contains a unique projection $e$, say, in which case $a^{+}=a^{*}$ for every $a \in T$ and $T$ is contained in $e \mathbb{H}$. Conversely, it is well known that any $\mathbb{H}$-class that contains a projection is a maximal submonoid.

The structural aspects, at least, of the following theorem are presumably folklore and date back, in the case of ample semigroups and beyond, to the work of Fountain [8]. That every strong semilattice of monoids is a restriction semigroup may be explicitly found in the thesis of Cornock [3, Section 1.6].

As a result of the following theorem, the class $\mathbf{S M}$ of all restriction semigroups that are semilattices of monoids is the subvariety $\mathbf{S L} \vee \mathbf{M}$ of $\mathbf{R}$. This theorem is a direct analogue of Result 1.7(2).

THEOREM 2.6 The following are equivalent for a restriction semigroup $S$ :

(i) $S \in \mathbf{S L} \vee \mathbf{M}$;

(ii) $S$ satisfies $x^{+}=x^{*}$;

(iii) $\mathbb{L}=\mathbb{R}(=\mathbb{H})$;

(iv) $S$ is a semilattice of monoids;

(v) $S$ is a union of monoids;

(vi) $S / \mu \in \mathbf{S L}$;

(vii) $P_{S}$ is central in $S$;

(viii) $S$ satisfies $(x y)^{+}=x^{+} y^{+}$;

(ix) $S$ is a strong semilattice of monoids. join.

Proof. (i) $\Rightarrow$ (ii). The stated identity is satisfied in $\mathbf{S L}$ and in $\mathbf{M}$ and therefore in their (ii) $\Rightarrow$ (iii). This is immediate from the definitions. 
(iii) $\Rightarrow$ (iv). Assuming $\mathbb{L}=\mathbb{R}$, then $\mathbb{H}$ is a (biunary) congruence. Moreover, since $a \mathbb{R} a^{+}$, $S / \mathbb{H}$ is a semilattice (regarded as a restriction semigroup). Each $\mathbb{H}$-class contains a unique member of $P_{S}$, since that is true of $\mathbb{R}$, and that projection must be $a^{+}=a^{*}$ for each $a$ in the class, so that the projection is its identity.

(iv) $\Rightarrow(\mathrm{v})$ is clear. That $(\mathrm{v}) \Rightarrow$ (ii) follows from the remarks in the second paragraph of this subsection.

(iv) $\Rightarrow$ (vi). If $S$ is a semilattice of monoids, denote by $\rho$ the induced semilattice congruence. Then $\rho$ is $P$-separating and so contained in $\mu$. But then $S / \mu$ is isomorphic to a quotient of $S / \rho$ and so belongs to SL.

(vi) $\Rightarrow$ (i). This is immediate from Proposition 2.1.

(ii) $\Rightarrow$ (vii). Let $e \in P_{S}$ and $a \in S$. Using (ii), $(a e)^{+}=(a e)^{*}=a^{*} e=e a^{+}$, whence $a e=(a e)^{+} a=e a$.

(vii) $\Rightarrow$ (viii). Let $a, b \in S$. Then $(a b)^{+}=\left(a b^{+}\right)^{+}=\left(b^{+} a\right)^{+}=b^{+} a^{+}=a^{+} b^{+}$.

(viii) $\Rightarrow$ (vii). Let $e \in P_{S}$ and $a \in S$. Then $a e=(a e)^{+} a=a^{+} e a=e a$.

(vii) $\Rightarrow$ (ii). Let $a \in S$. Using (vii), $a^{+}=\left(a a^{*}\right)^{+}=\left(a^{*} a\right)^{+}=a^{*} a^{+}$, that is, $a^{+} \leq a^{*}$. Equality follows by duality.

(iii) $\Rightarrow$ (ix). According to the proof of (iii) $\Rightarrow$ (iv), $S$ is the semilattice $Y=S / \mathbb{H}$ of the monoids $S_{e}=e \mathbb{H}, e \in P_{S}$. Let $e, g \in P_{S}, e \geq g$. Define $\phi_{e g}: S_{e} \rightarrow S_{g}$ by $a \phi_{e g}=g a$. Since by the previous implications, $P_{S}$ is central in $S, \phi_{e g}$ is a (biunary) homomorphism. The system thus defined is clearly transitive and for $a \in S_{e}, b \in S_{f}, a b=(e f) a b=(e f) a(e f) b$, as required. $\square$

Clearly, any restriction semigroup that is a semilattice of monoids as a restriction semigroup is also a semilattice of monoids as a 'plain' semigroup. It has the additional property that the identity elements of the monoids form a subsemilattice, which is not true for semilattices of monoids in general (for example, let $M$ be any monoid with a zero element and adjoin an element $e$ such that $e m=m e=0$ for all $m \in M$ ). We now show that the converse holds.

PROPOSITION 2.7 Let $S$ be any semigroup that is a semilattice $Y$ of monoids $S_{\alpha}, \alpha \in Y$, the identity elements of which form a subsemilattice of $S$. For $a \in S$, let both $a^{+}$and $a^{*}$ be the identity element of the submonoid $S_{\alpha}$ to which a belongs. Then $\left(S,{ }^{+},{ }^{*}\right)$ is a restriction semigroup that is a semilattice of monoids, in that context.

Proof. The axioms for a restriction semigroup are routinely verified, $x^{+} y^{+}=y^{+} x^{+}$following from the additional assumption on $S$. Since $Y$ itself becomes a restriction semigroup by putting $\alpha^{+}=\alpha^{*}=\alpha$, the additional requirements in that context are also satisfied.

The next theorem is a direct analogue of Result 1.7(3).

THEOREM 2.8 The sublattice $\mathcal{L}(\mathbf{S M})=\mathcal{L}(\mathbf{S L} \vee \mathbf{M})$ of $\mathcal{L}(\mathbf{R})$ is isomorphic to the direct product of the two-element lattice $\mathcal{L}(\mathbf{S L})$ and the lattice $\mathcal{L}(\mathbf{M})$, under the map $\mathbf{V} \mapsto(\mathbf{V} \cap \mathbf{S L}) \vee$ $(\mathbf{V} \cap \mathbf{M})$. If $\mathbf{V}$ is not simply a variety of monoids, then it consists of all (strong) semilattices of monoids from $\mathbf{V} \cap \mathbf{M}$. 
Proof. According to Theorem 2.3, the map $\mathbf{V} \longrightarrow \mathbf{V} \cap \mathbf{M}$ is a homomorphism, which is clearly surjective. Likewise, the map $\mathbf{V} \longrightarrow \mathbf{V} \cap \mathbf{S L}$ is a surjective morphism. (Since $\mathbf{S L}$ is an atom in the lattice of varieties, the only potential nontrivial case to consider is when $\mathbf{V}, \mathbf{W}$ are varieties such that $\mathbf{V} \cap \mathbf{S L}=\mathbf{W} \cap \mathbf{S L}=\mathbf{T}$; but then $\mathbf{V}$ and $\mathbf{W}$ are varieties of monoids and thus so is $\mathbf{V} \vee \mathbf{W}$.)

Let $\mathbf{V} \in \mathcal{L}(\mathbf{S M})$, with $\mathbf{V} \notin \mathbf{M}$. Thus $\mathbf{S L} \subseteq \mathbf{V}$. We must show that $\mathbf{V}=\mathbf{S L} \vee \mathbf{N}$, where $\mathbf{N}=\mathbf{V} \cap \mathbf{M}$. Now by Theorem 2.6, $S$ is (isomorphic to) a strong semilattice of monoids $S_{e}$, $e \in P_{S}$, where each of these (sub)monoids belongs to $\mathbf{N}$. The required outcome follows from a rather straightforward modification of the standard semigroup-theoretic arguments, cf [14, Proposition 4.6.11], so we omit the details.

\subsection{Some 'forbidden' restriction semigroups}

We study the basic properties of three restriction semigroups that in the next subsection will be shown to characterize, by their exclusion, varieties consisting of semilattices of monoids. They correspond, as we shall see there, to three possible ways in which that can occur: it must contain a restriction semigroup $S$ that possesses an element $a$ for which $a^{+} \neq a^{*}$, so that either $a^{+}>a^{*}, a^{+}<a^{*}$, or $a^{+}$and $a^{*}$ are incomparable. Although these examples have appeared in the literature, their importance does not seem to have been recognized.

These three examples derive from either the bicyclic semigroup $B$ or the five-element Brandt semigroup $B_{2}$. Those semigroups, which were introduced and briefly described in Subsection 1.1 as inverse semigroups, will now be treated as restriction semigroups. The examples we introduce are not themselves inverse semigroups, however.

Regarding $B$ as a restriction semigroup, and recalling the notation $e_{m}=b^{m} a^{m}, m \geq 0$, $\left(b^{m} a^{n}\right)^{+}=e_{m}$, for all $n \geq 0$, and $\left(b^{m} a^{n}\right)^{*}=e_{n}$, for all $m \geq 0$. Thus $P_{B}=E_{B}=\left\{e_{m}: m \geq 0\right\}$.

Let $B^{+}$be the 'upper triangle' of $B:\left\{b^{m} a^{n} \in B: n \geq m\right\}$. As a full subsemigroup of $B, B^{+}$is a restriction semigroup (in fact, it is ample) under the induced operations. The members of $B^{+}$can, alternatively, be uniquely represented in the form $\left(a^{m}\right)^{*} a^{k}$, where $m, k \geq 0$. Therefore $B^{+}$is indeed generated by $a$, as a restriction semigroup. Under that representation, $\left(\left(a^{m}\right)^{*} a^{k}\right)^{+}=\left(a^{m}\right)^{*}$ and $\left(\left(a^{m}\right)^{*} a^{k}\right)^{*}=\left(a^{m+k}\right)^{*}$. It is also clear that $B^{+}$is the complete inverse image, under the homomorphism onto $Z$, of the nonnegative integers. In fact, the congruence so induced on $B^{+}$is precisely the least monoid congruence $\sigma$. That is, $\left(a^{m}\right)^{*} a^{k} \sigma\left(a^{n}\right)^{*} a^{\ell}$ if and only if $k=\ell$ or, in the original notation, $b^{m} a^{n} \sigma b^{k} a^{\ell}$ if and only if $n-m=\ell-k$.

The semigroup $B^{+}$and its dual were studied by Makanjuola and Umar [20], who first showed they are ample. Descalço and Ruškuc [4] made a general study of the subsemigroups of $B$, regarded as a 'plain' semigroup; Descalço and Higgins [5] went on to study those subsemigroups that are 'abundant', which in our context means ample and, therefore, restriction semigroups.

PROPOSITION 2.9 Regarding $B^{+}$as a restriction semigroup:

(i) it satisfies the identity $x^{+} \geq x^{*}$;

(ii) it is presented by $\left\langle a \mid a^{+} \geq a^{*}\right\rangle$; 
(iii) any of its proper congruences identifies all its projections, so that its nontrivial homomorphic images are cyclic monoids (regarded as restriction semigroups);

(iv) it is proper.

Proof. (i) This is immediate from the earlier calculations: $\left(b^{m} a^{n}\right)^{+}=e_{m},\left(b^{m} a^{n}\right)^{*}=e_{n}$, and $n \geq m$.

(ii) Temporarily, put $P=\left\langle a \mid a^{+} \geq a^{*}\right\rangle$. Refer to the description of $F \mathbf{R}_{x}$ in Result 1.5. Mapping $x$ to $a, F \mathbf{R}_{x}$ maps onto $P$, so the elements of $P$ are expressible in the form $\left(a^{m}\right)^{*} a^{k}\left(a^{n}\right)^{+}$. From $a^{+} \geq a^{*}$ it follows that $a=a a^{*}=a a^{+} a^{*}=a a^{+}$. Thus $a^{+}$is an identity element of $P$. Further, $a^{+}=\left(a a^{+}\right)^{+}=\left(a^{2}\right)^{+}$and, by induction, $a^{+}=\left(a^{n}\right)^{+}$for every $n \geq 1$. Hence, the elements of $P$ are expressible as $\left(a^{m}\right)^{*} a^{k}$. Since $B^{+}$satisfies the defining relation and these elements are distinct therein, $B^{+} \cong P$.

(iii) Let $\rho$ be a congruence on $B^{+}$and suppose that $\left(a^{m}\right)^{*} a^{k} \rho\left(a^{n}\right)^{*} a^{\ell}$, using the alternative representation above. Then since $\rho$ respects the two unary operations, $\left(a^{m}\right)^{*} \rho\left(a^{n}\right)^{*}$ and $\left(a^{m+k}\right)^{*} \rho\left(a^{n+\ell}\right)^{*}$. It suffices, therefore, to show that if there exist $i<j$ such that $\left(a^{i}\right)^{*} \rho\left(a^{j}\right)^{*}$ then $\rho$ identifies all the projections. Under that hypothesis, since the projections are linearly ordered, $\left(a^{i}\right)^{*} \rho\left(a^{i+1}\right)^{*}$ and so $\left(a^{i}\right)^{*} \rho\left(a^{k}\right)^{*}$ for all $k>i$. In particular, $\left(a^{i}\right)^{*} \rho\left(a^{2 i}\right)^{*}$. Then, using the identities for right restriction semigroups, $\left(a^{i}\right)^{*} a^{i}=a^{i}\left(\left(a^{i}\right)^{*} a^{i}\right)^{*}=a^{i}\left(a^{2 i}\right)^{*} \rho a^{i}\left(a^{i}\right)^{*}=$ $a^{i}$. Since $a^{+}=\left(a^{i}\right)^{+}$and $\left(\left(a^{i}\right)^{*} a^{i}\right)^{+} \leq\left(a^{i}\right)^{*} \leq a^{*}$, it follows that $a^{+} \rho \leq a^{*} \rho$, that is $a^{+} \rho=a^{*} \rho$, in which case $a^{+} \rho=\left(a^{m}\right)^{*} \rho$ for all $m \geq 1$ and $B^{+} / \rho$ is a monoid.

(iii) This is immediate from the calculations prior to this proposition.

The second exceptional semigroup, $B^{-}$, is the dual $B^{+}$in the sense stated during the definition of right restriction semigroups in Section 1. Concretely, $B^{-}$is the 'lower triangle' in $B:\left\{b^{m} a^{n} \in B: n \leq m\right\}$, In that case, $b^{*}$ is the identity element of $B^{-}$. All the properties that we shall need of $B^{-}$follow from those of $B^{+}$, by duality. In particular, it satisfies the identity $x^{+} \leq x^{*}$.

The third semigroup, $B_{0}$, is the restriction subsemigroup of the five-element Brandt semigroup $B_{2}$ that is generated by $a$. (We follow the terminology of [18]. In [6] it was denoted $S(4,21)$.) Regarded as a restriction semigroup, $B_{2}=\left\{a, b, a^{+}=b^{*}, a^{*}=b^{+}, 0\right\}$. Thus $B_{0}=\left\{a, a^{+}, a^{*}, 0\right\}$, where $a^{+}$and $a^{*}$ are incomparable projections. Observe that the only pairwise products that do not yield 0 are $a^{+} a^{+}, a^{*} a^{*}, a^{+} a$ and $a a^{*}$. Edmunds [6] provided a set of defining identities for the semigroup variety generated by $B_{0}$, among which are the last two in the next statement. The first identity is straightforwardly verified (and is, in fact, satisfied in $B_{2}$ ).

LEMMA 2.10 The restriction semigroup $B_{0}$ satisfies the identities $x^{2}=x^{+} x^{*}, x^{3}=x^{2}$ and $x y x=x^{2} y^{2}$.

The following will prove useful in several circumstances.

PROPOSITION 2.11 Any proper restriction semigroup $S$ such that $S / \sigma$ is commutative satisfies the identity $(y x)^{+} x y=(x y)^{+} y x$. In particular, it is satisfied in the free monogenic restriction semigroup $F \mathbf{R}_{x}$ and thus in $B^{+}, B^{-}$and $B_{0}$ (even though $B_{0}$ is not itself proper). 
Proof. Since $S / \sigma$ is commutative, $(y x)^{+} x y \sigma(x y)^{+} y x$. But $\left((y x)^{+} x y\right)^{+}=\left((y x)^{+}(x y)^{+}\right)^{+}=$ $(y x)^{+}(x y)^{+}$and, similarly, $\left((x y)^{+} y x\right)^{+}=(x y)^{+}(y x)^{+}$. So $(y x)^{+} x y \mathbb{R}(x y)^{+} y x$ and equality then follows from properness.

\subsection{Minimal varieties of restriction semigroups that do not consist of semi- lattices of monoids}

According to Result 1.7(4), there is a unique variety of inverse semigroups, minimal with respect to the property that it does not consist of semilattices of monoids: that generated by $B_{2}$. The key to the analogue for restriction semigroups is the following.

LEMMA 2.12 Let $S$ be a restriction semigroup and $a \in S$. Either (i) $a^{+}>a^{*}$, in which case a generates a semigroup isomorphic to $B^{+}$, or (ii) $a^{+}<a^{*}$, in which case a generates a semigroup isomorphic to $B^{-}$, or (iii) $a^{+} \| a^{*}$, in which case $B_{0}$ divides $S$, or (iv) $a^{+}=a^{*}$, in which case a belongs to a submonoid of $S$.

Proof. Let $T$ be the restriction subsemigroup generated by $a$. In case (i), $T$ is a homomorphic image of $B^{+}$, by virtue of Proposition 2.9(ii). But since $T$ is not a monoid, by (iii) of the same proposition it must be isomorphic to $B^{+}$. The case (ii) is dual.

In case (iii), consider once again the homomorphism from $F \mathbf{R}_{x}$ upon $T$ that maps $x$ to $a$. Within $F \mathbf{R}_{x}$, the complement $I$ of $\left\{x, x^{+}, x^{*}\right\}$ constitutes a restriction-ideal. In the notation of Result 1.5, $I=\left\{\left(x^{m}\right)^{*} x^{k}\left(x^{n}\right)^{+}: m+k+n \geq 2\right\}$. The image $K$ of $I$ in $T$ is again a restrictionideal. We show that it is, likewise, the complement of $\left\{a, a^{+}, a^{*}\right\}$. Since if $a \in K$, then $a^{+} \in K$, it suffices, by duality, to show that $a^{+} \notin K$. If not, $a^{+}=\left(a^{m}\right)^{*}\left(a^{n}\right)^{+}$, for some $m, n \geq 0$, $m+n \geq 2$. If $m \neq 0$ then $a^{+} \leq\left(a^{m}\right)^{*} \leq a^{*}$. So $m=0, n \geq 2$ and $a^{+} \leq\left(a^{n}\right)^{+} \leq\left(a^{2}\right)^{+}$, in which case $a^{+}=\left(a^{2}\right)^{+}$and $a=\left(a^{2}\right)^{+} a=\left(a a^{+}\right)^{+} a=a a^{+}$. But this yields the contradiction $a^{*} \leq a^{+}$.

The Rees factor semigroup $T / K$ is therefore isomorphic to $B_{0}$.

In case (iv), $a$ belongs to the submonoid $e \mathbb{H}$, where $e=a^{+}=a^{*}$.

Denote by $\mathbf{B}^{+}, \mathbf{B}^{-}$and $\mathbf{B}_{0}$ the varieties of restriction semigroups generated by $B^{+}, B^{-}$and $B_{0}$, respectively. The main result of this section is the following.

THEOREM 2.13 (1) Any variety of restriction semigroups that does not consist of semilattices of monoids contains either $B^{+}, B^{-}$or $B_{0}$.

(2) The varieties $\mathbf{B}^{+}, \mathbf{B}^{-}$and $\mathbf{B}_{0}$ are the three varieties minimal with respect to not being contained in $\mathbf{S M}$.

Proof. (1) Let $\mathbf{V}$ be such a variety. As remarked at the beginning of the previous subsection, $\mathbf{V}$ must contain a restriction semigroup $S$ that contains an element $a$ such that either $a^{+}>a^{*}$, $a^{+}<a^{*}$, or $a^{+}$and $a^{*}$ are incomparable. Lemma 2.12 now yields the desired conclusion.

(2) Clearly none of the three varieties is contained in SM. In view of (1), to prove each is minimal with respect to that property it suffices to show that none of the three varieties 
contains the generating semigroup of either of the others. That this is true for $\mathbf{B}^{+}$is immediate from the fact that it satisfies $x^{+} \geq x^{*}$, and similarly for $\mathbf{B}^{-}$.

To show it is true for $\mathbf{B}_{0}$, we first show that $\mathbf{B}_{0} \cap \mathbf{M}=\mathbf{T}$. Any restriction monoid in $\mathbf{B}_{0}$ satisfies $x^{2}=x^{+} x^{*}=1$, from which the identity $x^{3}=x^{2}$ implies $x=1$. Since $\mathbf{B}^{+}$and $\mathbf{B}^{-}$each contain the infinite cyclic monoid, neither is contained in $\mathbf{B}_{0}$.

The relationships among these varieties are now investigated further. Recall that $\mathbf{M C}$ denotes the variety of commutative monoids.

PROPOSITION 2.14 (1) $\mathbf{B}^{+}$and $\mathbf{B}^{-}$each cover $\mathbf{S L} \vee \mathbf{M C}$ and thus $\mathbf{B}^{+} \cap \mathbf{B}^{-}=\mathbf{S L} \vee \mathbf{M C}$.

(2) $\mathbf{B}_{0}$ covers $\mathbf{S L}$.

Proof. (1) By Proposition 2.11, the identity $(y x)^{+} x y=(x y)^{+} y x$ is satisfied in $\mathbf{B}^{+}$, so any monoid in $\mathbf{B}^{+}$is commutative. But $\mathbf{B}^{+}$contains the infinite cyclic monoid and therefore all commutative monoids. So $\mathbf{B}^{+} \cap \mathbf{M}=\mathbf{M C}$ and, since $\mathbf{S L} \subset \mathbf{B}^{+}$, applying Theorem 2.8 yields $\mathbf{B}^{+} \cap \mathbf{S M}=\mathbf{S L} \vee \mathbf{M C}$. The covering property follows from Theorem 2.13(2).

By duality, and since $\mathbf{B}^{+}$and $\mathbf{B}^{-}$are incomparable, their intersection is therefore $\mathbf{S L} \vee \mathbf{M C}$.

(2) That $\mathbf{B}_{0} \cap \mathbf{M}=\mathbf{T}$ was shown in the course of proving Theorem 2.13(2). Then, again applying Theorem 2.8, $\mathbf{B}_{0} \cap \mathbf{S M}=\mathbf{S L}$ and the covering is obvious.

PROPOSITION 2.15 (1) $\mathbf{B}^{+} \vee \mathbf{B}^{-}=\mathbf{V}\left(F \mathbf{R}_{x}\right)$; therefore (2) $\mathbf{B}^{+} \vee \mathbf{B}^{-}$contains $\mathbf{B}_{0}$.

Proof. (1) See Result 1.5 for the requisite properties of $F \mathbf{R}_{x}$. Since $B^{+}$and $B^{-}$are monogenic, they each belong to $\mathbf{V}\left(F \mathbf{R}_{x}\right)$. To show $F \mathbf{R}_{x} \in \mathbf{B}^{+} \vee \mathbf{B}^{-}$, let $R$ denote the restriction subsemigroup of $B^{-} \times B^{+}$generated by the element $r=(b, a)$. The map $x \mapsto r$ extends to a homomorphism $\phi: F \mathbf{R}_{x} \longrightarrow R$. Since $\mathbb{H}=\iota$ on $F \mathbf{R}_{x}$, it suffices to show that $\phi$ separates the projections of $F \mathbf{R}_{x}$, for then it must be an isomorphism. Evaluating the projection $\left(x^{m}\right)^{*}\left(x^{n}\right)^{+}$(where $\left.m, n \geq 0, m+n \geq 1\right)$ in $R$, we obtain $\left(\left(b^{m}\right)^{*},\left(a^{m}\right)^{*}\right)\left(\left(b^{n}\right)^{+},\left(a^{n}\right)^{+}\right)=$ $\left(1,\left(a^{m}\right)^{*}\right)\left(\left(b^{n}\right)^{+}, 1\right)=\left(\left(b^{n}\right)^{+},\left(a^{m}\right)^{*}\right)$. Since the idempotents $\left(b^{n}\right)^{+}$are distinct in $B^{-}$and likewise the idempotents $\left(a^{m}\right)^{*}$ are distinct in $B^{+}$, the same is true for the specified idempotents in $R$, as claimed.

(2) Since $B_{0}$ is monogenic, this follows from (1).

COROLLARY 2.16 Any variety of restriction semigroups that does not contain $B_{0}$ is contained in either the variety defined by $x^{+} \geq x^{*}$ or the variety defined by $x^{+} \leq x^{*}$.

Proof. From (2) of the proposition, it follows from Lemma 2.12 that if $B_{0} \notin \mathbf{V}$, then either $a^{+} \geq a^{*}$ for all $a \in S$ and for all $S \in \mathbf{V}$, or the dual statement holds.

PROPOSITION 2.17 (1) The bicyclic semigroup $B$ is not contained in the join of the varieties defined by $x^{+} \geq x^{*}$ and $x^{+} \leq x^{*}$, respectively;

(2) thus $\mathbf{B}^{+} \vee \mathbf{B}^{-} \subsetneq \mathbf{V}(B)$. 
Proof. (1) It suffices to show that the identity $(x y)^{*} x^{+} y^{+} \leq\left(x^{+} y\right)^{*}$ is a consequence of each of the identities $x^{+} \geq x^{*}$ and $x^{+} \leq x^{*}$, but is not satisfied in $B$. From $x^{+} \geq x^{*}$ it follows, using (the dual of) Lemma 1.1 that $(x y)^{*}=\left(x^{*} y\right)^{*}=\left(x^{*}\left(x^{+} y\right)\right)^{*} \leq\left(x^{+} y\right)^{*}$; from $x^{+} \leq x^{*}$ it follows that $x^{+} y^{+}=\left(x^{+} y\right)^{+} \leq\left(x^{+} y\right)^{*}$. In either case, the stated inequality holds.

Now substitute $x=b$ and $y=a$ from $B$. Here $x^{+}=b^{+}=a^{*}, y^{+}=a^{+}=1,(x y)^{*}=(b a)^{*}=$ $b a=a^{*}$, so the left hand side yields $a^{*}$. But $\left(x^{+} y\right)^{*}=\left(a^{*} a\right)^{*}=\left(a^{2}\right)^{*}$ and $a^{*}>\left(a^{2}\right)^{*}$ in $B$.

(2) Containment follows from the fact that $B^{+}$and $B^{-}$are restriction subsemigroups of $B$. Strictness follows from (1) and Proposition 2.9(i) and its dual.

PROPOSITION 2.18 $B^{-} \notin \mathbf{B}_{0} \vee \mathbf{B}^{+}$and $B^{+} \notin \mathbf{B}_{0} \vee \mathbf{B}^{-}$.

Proof. It suffices to prove the former statement. The identity $x^{+} \geq\left(x^{2}\right)^{*}$ holds in $B_{0}$, as a consequence of the first identity specified in Lemma 2.10. It also holds in $B^{+}$since from $x^{+} \geq x^{*}$ we obtain $x^{*}=\left(x^{+} x\right)^{*} \geq\left(x^{*} x\right)^{*}=\left(x^{2}\right)^{*}$. However this identity fails in $B^{-}$because $b^{+}=b a<\left(b^{2}\right)^{*}=a^{2} b^{2}=1$.

The varieties generated by $B_{0}$ and $B_{2}$, and their joins with $\mathbf{M}$, will be studied in depth in the sequel [17].

\section{$3 \quad$ Varieties of left restriction semigroups}

\subsection{Joins and meets with $\mathrm{M}$}

The proofs of the two theorems on the corresponding topic for restriction semigroups need almost no modification to yield their analogues for left restriction semigroups. (Note that the defining property of $\mu$, and its description, must be appropriately modified in the proof of Theorem 2.1; also note that the proof of Lemma 2.2 was written in such a way that it remains valid in the one-sided situation.)

THEOREM 3.1 If $\mathbf{V} \in \mathcal{L}(\mathbf{L R})$, then $\mathbf{V} \vee \mathbf{M}=\{S \in \mathbf{L R}: S / \mu \in \mathbf{V}\}$. Hence the map $\mathbf{V} \longrightarrow \mathbf{V} \vee \mathbf{M}$ is a complete lattice homomorphism.

If $\mathbf{V}$ is defined by the identities $u_{i}=v_{i}, i \in I$, then $\mathbf{V} \vee \mathbf{M}$ is defined by the identities $u_{i}^{+}=v_{i}^{+}$and $\left(u_{i} x\right)^{+}=\left(v_{i} x\right)^{+}, i \in I$, where $x$ is a letter distinct from any in the original set of identities.

THEOREM 3.2 The map $\mathbf{V} \longrightarrow \mathbf{V} \cap \mathbf{M}, \mathbf{V} \in \mathcal{L}(\mathbf{R})$ is a homomorphism.

Analysis of the proof that $\mathbf{M}$ does not separate $\mathcal{L}(\mathbf{R})$ (Proposition 2.5) reveals that the operation * was never used. Thus, when all the semigroups considered therein are instead regarded as left restriction semigroups, the argument also proves the analogous result.

PROPOSITION 3.3 The variety of monoids does not separate the lattice LR.

Once again, the example used in the proof could be considerably simplified to provide the same outcome. 


\subsection{Varieties of left restriction semigroups that consist of semilattices of monoids}

Referring to Subsection 2.2, we shall use the same notation, SM, to denote here the class of left restriction semigroups that are semilattices of monoids, with the analogous constraints on the components. As a result of the next theorem, SM is a subvariety of $\mathbf{L R}$.

Once again, it is clear that in such a semigroup the identity elements of the component monoids form a semilattice. Thus by Proposition 2.7, every such semigroup is in fact a restriction semigroup and Theorem 2.6 can be applied, in the cases that do not make reference to the second unary operation.

THEOREM 3.4 The following are equivalent for a left restriction semigroup $S$ :

(i) $S \in \mathbf{S L} \vee \mathbf{M}$;

(ii) $S$ satisfies $x y^{+}=y^{+} x$, that is, $P_{S}$ is central in $S$;

(iii) $S$ satisfies $(x y)^{+}=x^{+} y^{+}$;

(iv) $S$ becomes a restriction semigroup under the assignment $a^{*}=a^{+}, a \in S$.

(v) $S$ is a semilattice of monoids;

(vi) $S$ is a strong semilattice of monoids.

Proof. The identity in (ii) is satisfied in SL and in $\mathbf{M}$ and so in their join. Therefore (ii) follows from (i). The equivalence of (ii) and (iii) uses the same argument as that of (vii) and (viii) in Theorem 2.6. That (ii) implies (iv) is immediate from the duality that yields the identities for right restriction semigroups. Now the equivalence of (iv), (v) and (vi), and the implication (v) $\Rightarrow$ (i) follow from Theorem 2.6 itself.

The following is then obtained from Theorem 2.8. Again, it is a direct analogue of Result 1.7(3).

THEOREM 3.5 The sublattice $\mathcal{L}(\mathbf{S M})$ of $\mathcal{L}(\mathbf{L R})$ is isomorphic to the direct product of the two-element lattice $\mathcal{L}(\mathbf{S L})$ and the lattice $\mathcal{L}(\mathbf{M})$, under the map $\mathbf{V} \mapsto(\mathbf{V} \cap \mathbf{S L}) \vee(\mathbf{V} \cap \mathbf{M})$. If $\mathbf{V}$ is not simply a variety of monoids, then it consists of all (strong) semilattices of monoids from $\mathbf{V} \cap \mathbf{M}$.

\subsection{Varieties of left restriction semigroups that consist of unions of monoids}

It was seen in Theorem 2.6 that a restriction semigroup that is a union of submonoids is necessarily a semilattice of monoids. The semigroup $B^{+}$, regarded as a left restriction semigroup, illustrates that this is no longer true in the one-sided case: see Proposition 3.7 below. First we clarify the form of the maximal submonoids in left restriction semigroups. 
LEMMA 3.6 The maximal submonoids of a left restriction semigroup have the form $M_{e}=$ $e \mathbb{R} \cap e S e, e \in P_{S}$. Alternatively, $M_{e}=\left\{a \in S: a^{+}=e, a e=a\right\}$.

Proof. Let $e \in P_{S}$. If $a, b \in e \mathbb{R} \cap e S e$, then $(a b)^{+}=\left(a b^{+}\right)^{+}=(a e)^{+}=a^{+}=e$, so $M_{e}=e \mathbb{R} \cap e S e$ is a subsemigroup that is clearly a submonoid. Conversely, if $M$ is any submonoid of $S$, with projection $e$, then clearly $M \subseteq M_{e}$. The second statement is just a reformulation of the first.

A plentiful source of examples for this subsection is found among the subsemigroups of the left restriction semigroup $B^{+}$, which was studied as a restriction semigroup in Subsection 2.3.

PROPOSITION 3.7 Regarded as a left restriction semigroup, $B^{+}$is the union of its infinite cyclic submonoids $M_{e_{m}}=\left\{b^{m} a^{n}: n \geq m\right\}, m \geq 0$. Every left restriction subsemigroup of $B^{+}$ is a union of monoids. Such a subsemigroup is not a semilattice of monoids unless it is either (a) a subsemilattice, (b) a submonoid or (c) the union of a nontrivial submonoid of some $M_{e_{m}}$ with a set of projections $e_{k}, k<m$.

Proof. For a given $m \geq 0$, direct calculation shows that $M_{e_{m}}=\left\{b^{m} a^{n}: n \geq m\right\}$. Clearly $B^{+}$is the union of these submonoids. Note that $M_{e_{m}}$ is generated, as a monoid, by the element $a_{m}=e_{m} a=b^{m} a^{m+1}$ and so it is infinite cyclic. Now if $T$ is a left restriction subsemigroup of $B^{+}$that is not a subsemilattice, it contains some element $c=b^{m} a^{n}, m \geq 0, n>m$; if, further, $T$ does not satisfy (c), then it contains a projection $e_{k}, k>m$. Now by taking powers of $c$, if necessary, it may be assumed that $n>k$ and direct calculation in $B$ then shows that $\left(c e_{k}\right)^{+}=c^{+}=e_{m}$ but $\left(e_{k} c\right)^{+}=e_{k}$. So the projections of $T$ are not central and Theorem 3.4 applies.

As a result of the next theorem, the class $\mathbf{U M}$ of left restriction semigroups that are unions of monoids is a subvariety of $\mathbf{L R}$.

THEOREM 3.8 The following are equivalent for a left restriction semigroup $S$ :

(i) $S$ is a union of monoids;

(ii) $S$ satisfies $x x^{+}=x$;

(iii) $S$ satisfies $x^{+}=\left(x^{2}\right)^{+}$;

(iv) for each $a \in S,\left\{a^{+}\right\} \cup\left\{a^{i}: i \geq 1\right\}$, is a submonoid, which is the left restriction subsemigroup generated by a;

(v) each $\mathbb{R}$-class is a submonoid.

Proof. Clearly, if $S$ is a union of monoids, $a \in M_{a^{+}}$for any $a \in S$, so $a a^{+}=a$. The equivalence of (ii) and (iii) follows immediately from the equation $x x^{+}=\left(x x^{+}\right)^{+} x=\left(x^{2}\right)^{+} x$. By induction, the identity in (iii) implies satisfaction of $x^{+}=\left(x^{n}\right)^{+}$for all $n \geq 1$, from which 
(iv) follows. If (iv) holds, then (i) is clear. Finally, from (ii) it follows that if $a^{+}=b^{+}$, then $(a b)^{+}=\left(a b^{+}\right)^{+}=\left(a a^{+}\right)^{+}=a^{+}$, so that each $\mathbb{R}$-class is a subsemigroup, which is clearly then a submonoid of $S$. Once again, if (v) holds, (i) is clear.

The lattice $\mathcal{L}(\mathbf{U M})$ appears to be much more complicated in structure than $\mathcal{L}(\mathbf{S M})$ and warrants further study. In addition to determining the unique semigroup that discriminates varieties of semilattices of monoids from varieties of unions of monoids in general (Subsection 3.5), we will content ourselves with the following straightforward observations.

COROLLARY 3.9 Every left restriction semigroup that is a union of monoids has a proper cover of the same type. In fact all of its proper covers are unions of monoids.

Proof. By Result 1.2, every such semigroup has a proper cover, so it suffices to prove the second statement. We apply (iii) from the theorem. Suppose $T$ is a proper cover of the union of monoids $S$, via the homomorphism $\phi$, and let $a \in T$. Then $a^{+} \phi=\left(a^{2}\right)^{+} \phi$. Since $\phi$ separates projections, $a^{+}=\left(a^{2}\right)^{+}$.

We regard monoid identities over an alphabet $X$ as equalities of words in the free monoid $X^{*}$ on $X$. The content of such a word is the set of members of $X$ (possibly empty) that appear in the word. Regarded as identities of left restriction semigroups, 1 needs to be replaced by $x^{+}$ (where $x^{+}=y^{+}$is the identity defining monoids within the latter class of semigroups).

PROPOSITION 3.10 Let $u\left(x_{1}, \ldots, x_{m}\right)=v\left(y_{1}, \ldots, y_{n}\right)$ be a nontrivial monoid identity. Denote by $p$ the product of the terms $z^{+}$, over the union of the contents of the two words. Let $S \in \mathbf{U M}$. The following are equivalent:

1. $M_{e}$ satisfies the identity $u\left(x_{1}, \ldots, x_{m}\right)=v\left(y_{1}, \ldots, y_{n}\right)$ for all $e \in P_{S}$;

2. $S$ satisfies the identity $p u\left(p x_{1}, \ldots, p x_{m}\right)=p v\left(p y_{1}, \ldots, p y_{n}\right)$.

Let $\mathbf{N}$ be a variety of monoids. Then the class $\mathbf{U N}$ of (left restriction semigroups that are) unions of monoids from $\mathbf{N}$ is a subvariety of $\mathbf{U M}$, whose identities are obtained from those of $\mathbf{N}$ as above, and $\mathbf{U N}=\left\{S \in \mathbf{U M}: M_{e} \in \mathbf{N} \forall e \in P_{S}\right\}$.

Proof. If $S$ satisfies the second identity, then in any submonoid $T$ all the terms $z^{+}$evaluate to the identity of $T$ and so the original identity holds in $T$. Conversely, suppose each submonoid satisfies the original identity. If the second identity is evaluated in $S$, then the evaluation of $p$ in $S$ is a projection, each $\left(p x_{i}\right)^{+}=p x_{i}^{+}=p$ and, similarly, each $\left(p y_{j}\right)^{+}=p$. Assuming the first identity holds in $M_{p}$, the second therefore holds in $S$.

For instance, UMC is defined within $\mathbf{U M}$ by $\left(x^{+} y^{+} x\right)\left(x^{+} y^{+} y\right)=\left(x^{+} y^{+} y\right)\left(x^{+} y^{+} x\right)$, or just $y^{+} x y=x^{+} y x$, using the identity for $\mathbf{U M}$ given in Theorem 3.8(ii).

An interesting question arises from the following result of Cornock. A [left] restriction semigroup has a proper cover over a variety $\mathbf{N}$ of monoids if it has a proper cover $T$ such that $T / \sigma \in \mathbf{N}$. 
RESULT 3.11 [3, Theorems 9.6.2 and 10.3.4] For any variety $\mathbf{N}$ of monoids, the class $\widehat{\mathbf{N}}$ of left restriction semigroups having a proper cover over $\mathbf{N}$ forms a variety, defined by the set of identities $v^{+} u=u^{+} v$, where $u=v$ is a monoid identity satisfied in $\mathbf{N}$.

In light of Corollary 3.9, this result remains true within the confines of unions of monoids, that is, for the variety $\widehat{\mathbf{N}} \cap \mathbf{U M}$. It is easily seen that this variety is a subvariety of $\mathbf{U N}$ and the question arises as to whether the inclusion can be strict.

For example, notice that the identity $(y x)^{+} x y=(x y)^{+} y x$ is a consequence of $y^{+} x y=x^{+} y x$, so every member of $\mathbf{U M C}$ has a proper cover of the same type, that is, $\mathbf{U M C}=\widehat{\mathbf{M C}} \cap \mathbf{U M}$. Similarly, equality holds for the variety MSL of semilattice monoids.

Of interest in a different direction, in light of Proposition 3.3, is the question whether or not $\mathbf{M}$ separates the lattice $\mathcal{L}(\mathbf{U M})$.

\subsection{Some 'forbidden' left restriction semigroups}

We introduce and study the basic properties of several left restriction semigroups that will appear in the next two subsections. The inverse semigroups $B$ and $B_{2}$, together with the restriction subsemigroups $B^{+}, B^{-}$of $B$ and the restriction subsemigroup $B_{0}$ of $B_{2}$ that were introduced in Subsection 2.3, will now be treated as left restriction semigroups.

The first semigroup of note is the three-element left restriction subsemigroup $D=\left\{a, a^{+}, 0\right\}$ of $B_{0}$ and, ultimately, of $B_{2}$. We use the notation of [19]. (It was denoted $S(2,4)$ in the survey [6] of small semigroups.) The only pairwise products in $D$ that do not yield 0 are $a^{+} a$ and $a^{+} a^{+}$.

LEMMA 3.12 The left restriction semigroup D may be presented as such by $\left\langle a \mid a a^{+}=\left(a^{2}\right)^{+}\right\rangle$ and satisfies the identity $x x^{+}=\left(x^{2}\right)^{+}$. It is not a union of monoids.

Proof. As previously noted, $x x^{+}=\left(x^{2}\right)^{+} x$ in any left restriction semigroup, as a consequence of the left ample identity. Since $a^{2}=0$ in $D$, the relation $a a^{+}=\left(a^{2}\right)^{+}$is clear. Now if $S=\left\langle a \mid a a^{+}=\left(a^{2}\right)^{+}\right\rangle$, then $\left(a^{2}\right)^{+}=a a^{+}=\left(a^{2}\right)^{+} a,\left(a^{2}\right)^{+}=\left(a^{2}\right)^{+} a^{2}=a^{2}$ and, by induction, $\left(a^{2}\right)^{+}=a^{p}$ for all $p \geq 2$. According to Result 1.6, $S=\left\{\left(a^{p}\right)^{+} a^{q}: p \geq 1,0 \leq q \leq p\right\}$, where for $p \geq 2,\left(a^{p}\right)^{+} a^{q}=\left(a^{2}\right)^{+} a^{q}=\left(a^{2}\right)^{+}$. Since only $a^{+}$and $a$ remain to be considered, $|S| \leq 3$ and so $S \cong D$.

As just noted, the identity $x x^{+}=\left(x^{2}\right)^{+}$is satisfied when $x=a$. Otherwise, $x$ is a projection and it is clearly satisfied.

Since $a a^{+}=0, D$ is not a union of monoids.

The second semigroup of note is the left restriction subsemigroup $B_{01}^{+}=M_{e_{0}} \cup M_{e_{1}}$ of $B^{+}$and, ultimately, of $B$. It is routinely verified that $B_{01}^{+}$is indeed a subsemigroup. For convenience, let $c=e_{1} a$, the generator of $M_{e_{1}}$.

PROPOSITION 3.13 Regarding $B_{01}^{+}$as a left restriction semigroup:

(i) it is a union of monoids, but not a semilattice of monoids; 
(ii) it is presented by $\left\langle a, c \mid a c^{+}=a, c^{+} a=c\right\rangle$;

(iii) its $\mu$-classes are the sets $\left\{e_{0}\right\},\left\{a^{n}: n \geq 1\right\}$ and $M_{e_{1}}$;

(iv) it is proper, $b^{k} a^{\ell} \sigma b^{m} a^{n}$ if and only if $\ell-k=n-m$, and $B_{01}^{+} / \sigma$ is an infinite cyclic monoid;

(v) it belongs to $\mathbf{U M C}$ and so satisfies $y^{+} x y=x^{+} y x$.

Proof. (i) This is a special case of Proposition 3.7.

(ii) $M_{e_{0}}$ and $M_{e_{1}}$ are generated, respectively, by $a$ and $c$, so $B_{01}^{+}$is generated by $\{a, c\}$. The first equation holds because $c^{+}=e_{1}=b a$ and $a(b a)=a$, the second from the definition of $c$.

Now let $S$ be the left restriction semigroup presented as stated. Then $c^{+}=\left(c^{+} a\right)^{+}=c^{+} a^{+}$, that is, $c^{+} \leq a^{+}$. So $a a^{+}=\left(a c^{+}\right) a^{+}=a c^{+}=a$. As in the proof of Theorem 3.8, $\left(a^{n}\right)^{+}=a^{+}$ for $n \geq 1$ and so $a$ generates a submonoid, with identity $a^{+}$. Similarly, $c c^{+}=\left(c^{+} a\right) c^{+}=$ $c^{+}\left(a c^{+}\right)=c^{+} a=c$ and $c$ generates a submonoid, with identity $c^{+}$. Further, $a c=a\left(c^{+} a\right)=$ $\left(a c^{+}\right) a=a^{2}$ and so $a^{i} c^{j}=a^{i+j}$, for $i \geq 1, j \geq 0$. (However, $a^{0} c=a^{+} c=c$.) Similarly, $c a=\left(c c^{+}\right) a=c\left(c^{+} a\right)=c^{2}$ and so $c^{i} a^{j}=c^{i+j}$ for $i \geq 0, j \geq 1$. Thus $S$ is the union of the submonoids generated by $a$ and $c$.

Finally, the powers of $a$ and of $c$ are distinct in $B_{01}^{+}$and so the map from $S$ onto that semigroup is an isomorphism.

(iii) Recall that on an arbitrary left restriction semigroup $S, x \mu y$ if and only if $(x e)^{+}=$ $(y e)^{+}$for all $e \in P_{S}^{1}$. Since the only projections of $B_{01}^{+}$are the identity element $e_{0}$ and $e_{1}$, $x \mu y$ if and only if $\left(x e_{1}\right)^{+}=\left(y e_{1}\right)^{+}$. Thus $\mu$ identifies all the powers of $c$ with $c^{+}=e_{1}$; it also identifies all the positive powers of $a$ with $a$ itself, since $a^{i} c^{+}=a^{i}$ for all $i \geq 1$. But $\mu$ does not identify $a$ with $a^{+}$(for otherwise it also identifies $e_{1}=c^{+}=a^{+} c^{+}$with $a c^{+}=a$ and thus with $a^{+}=e_{0}$.)

(iv) This follows either by direct calculation or from observing that $\sigma$ is the restriction to $B_{01}^{+}$of the least group congruence on $B$ itself.

(v) This is a consequence of the comments following Proposition 3.10.

The third semigroup of note, denoted $L_{2}^{1}$, is obtained from the two-element left zero semigroup $L_{2}=\{e, f\}$ by adjoining an identity 1 , setting $e^{+}=e$ and $f^{+}=1^{+}=1$. It is routinely verified that it is a left restriction semigroup. Note that, when regarded as a 'plain' semigroup, $L_{2}^{1}$ is a monoid, but not when regarded as a left restriction semigroup.

PROPOSITION 3.14 The left restriction semigroup $L_{2}^{1}$

(i) is isomorphic to $B_{01}^{+} / \mu$;

(ii) is a union of monoids, but not a semilattice of monoids;

(iii) is presented by $\left\langle a, c \mid a c^{+}=a, c^{+} a=c, a^{2}=a, c^{+}=c\right\rangle$;

(iv) belongs to UMSL and so satisfies $y^{+} x y=x^{+} y x$. 
Proof. (i) Using (iii) of the previous proposition, identify $e_{1} \mu$ with $e, a \mu$ with $f$, and $e_{0} \mu$ with 1.

(ii) Because the projection $e$ is not central $(e f=e$, but $f e=f), L_{2}^{1}$ is not a semilattice of monoids. It is the union of the semilattice monoid $M_{1}=\{1, f\}$ and the trivial monoid $M_{e}$.

(iii) With $a=f$ and $c=e$, the relations are clearly satisfied. Now let $S$ be the left restriction semigroup so presented. As in the proof of Proposition 3.13(ii), $S$ is the union of the cyclic monoids generated by $a$ and $c$ and, applying the additional relations, $S$ consists at most of the three elements $a^{+}, a$, and $c$. Therefore $S \cong L_{2}^{1}$.

(iv) Clearly each submonoid is a semilattice and so commutative. The stated identity then follows as for $B_{01}^{+}$.

PROPOSITION 3.15 $B_{01}^{+}$is a subdirect product of $L_{2}^{1}$ and the infinite cyclic monoid.

Proof. According to Proposition 3.13(iv), $B_{01}^{+}$is proper and so, by Lemma 1.3, a subdirect product of $B_{01}^{+} / \mu$ and $B_{01}^{+} / \sigma$. The conclusion then follows from the quoted proposition, in combination with Proposition 3.14(i).

PROPOSITION 3.16 Any left restriction semigroup that is a union of monoids, but not a semilattice of monoids, contains as a left restriction subsemigroup a P-separating quotient of $B_{01}^{+}$.

Proof. Suppose $S$ is a union of monoids that is not a semilattice of monoids. Then by Theorem 3.4, $P_{S}$ is not central, so there exist $e \in P_{S}$ and $s \in S$ such that $a=s e \neq e s=c$. By Theorem 3.8, $a=a a^{+}$and $s=s s^{+}$. Now $a c^{+}=(s e)(e s)^{+}=s e s^{+}=s e=a$; and $c^{+} a=$ $(e s)^{+}(s e)=e s^{+} s e=e s e=e s e s^{+}=(e s)(e s)^{+}=e s=c$. The left restriction subsemigroup generated by $a$ and $c$ is therefore a quotient of $B_{01}^{+}$, by Proposition 3.13. Note that $a^{+} \neq c^{+}$ (otherwise $a=c^{+} a=c$ ), so the quotient is $P$-separating.

\subsection{Minimal varieties of left restriction semigroups that do not consist of unions of monoids}

Denote by $\mathbf{D}$ the variety generated by the semigroup $D$.

THEOREM 3.17 Any variety of left restriction semigroups that does not consist solely of unions of monoids contains $D$. Thus $\mathbf{D}$ is the smallest variety of left restriction semigroups with this property.

Proof. According to Theorem 3.8, a left restriction semigroup that is not a union of monoids contains a left restriction subsemigroup generated by an element $a$ such that $a a^{+} \neq a$. Thus any variety $\mathbf{V}$ that is not contained in $\mathbf{U M}$ contains a semigroup $S$ of this form. Further, there is a homomorphism $F \mathbf{L R} \mathbf{R}_{x} \longrightarrow S$, mapping $x$ to $a$.

The set $I_{2}=\left\{\left(x^{n}\right)^{+} x^{k}: n \geq 2,0 \leq k \leq n\right\}$ is clearly a ${ }^{+}$-ideal of $F \mathbf{L R}_{x} ; F \mathbf{L R}_{x} \backslash I_{2}=$ $\left\{x^{+}, x\right\}$. The image $K_{2}$ of $I_{2}$ in $S$ is again a ${ }^{+}$-ideal. If $K_{2}$ were to contain $a$, then it would contain $a^{+}$, and vice versa; but in that case $a^{+}=\left(a^{n}\right)^{+}$, for some $n \geq 2$, yielding the contradiction $a^{+}=\left(a^{2}\right)^{+}$. So $S \backslash K_{2}=\left\{a^{+}, a\right\}$ and $S / K_{2} \cong D$. 
PROPOSITION 3.18 D covers SL.

Proof. Since $D$ contains the two-element subsemilattice $\left\{0, a^{+}\right\}, \mathbf{D}$ contains SL. Now if $\mathbf{S L} \subseteq \mathbf{V} \subset \mathbf{D}$, then by Theorem 3.17, $\mathbf{V}$ consists of unions of monoids and therefore satisfies $x=x x^{+}$. By Lemma 3.12, $D$ satisfies the identity $x x^{+}=\left(x^{2}\right)^{+}$. Thus $\mathbf{V}$ satisfies $x=\left(x^{2}\right)^{+}$ and so consists of semilattices.

\subsection{Minimal varieties of left restriction semigroups that do not consist of semilattices of monoids}

Denote by $\mathbf{L}_{2}^{1}$ the variety generated by $L_{2}^{1}$.

THEOREM 3.19 Any variety of unions of monoids that does not consist solely of semilattices of monoids contains $L_{2}^{1}$. Thus $\mathbf{L}_{\mathbf{2}}^{\mathbf{1}}$ is the smallest subvariety of $\mathbf{U M}$ with this property.

Proof. According to Proposition 3.16, any such variety contains a $P$-separating quotient of $B_{01}^{+}$, and so contains $L_{2}^{1} \cong B_{01}^{+} / \mu$.

THEOREM 3.20 (1) Any variety of left restriction semigroups that does not consist of semilattices of monoids contains either $D$ or $L_{2}^{1}$.

(2) The varieties $\mathbf{D}$ and $\mathbf{L}_{2}^{1}$ are the two varieties minimal with respect to not being contained in $\mathbf{S M}$.

Proof. Clearly, since $\mathbf{L}_{\mathbf{2}}^{\mathbf{1}}$ consists of unions of monoids, it does not contain $\mathbf{D}$; the converse also holds for, by Proposition 3.18, the only unions of monoids in $\mathbf{D}$ are semilattices.

PROPOSITION 3.21 $\mathbf{L}_{2}^{1} \cap \mathbf{M}=\mathbf{M S L}$, the variety of monoids that consists of semilattices. Thus $\mathbf{L}_{2}^{1}$ covers $\mathbf{S L} \vee$ MSL.

Proof. The variety $\mathbf{L}_{\mathbf{2}}^{\mathbf{1}}$ satisfies $x^{2}=x$ and the identity stated in Proposition 3.14(iv), so $\mathbf{L}_{\mathbf{2}}^{\mathbf{1}} \cap \mathbf{M} \subseteq \mathbf{S L}$. Conversely, the variety of semilattice monoids is generated by the two-element semilattice monoid, which is a submonoid of $L_{2}^{1}$. So equality holds.

Now $P_{L_{2}^{1}}$ is a two-element semilattice (regarded in this case as a left restriction semigroup), so $\mathbf{S L} \subset \mathbf{L}_{\mathbf{2}}^{\mathbf{1}}$ and thus $\mathbf{S L} \vee \mathbf{M S L} \subset \mathbf{L}_{\mathbf{2}}^{\mathbf{1}}$. If $\mathbf{S L} \vee \mathbf{M S L} \subseteq \mathbf{V} \subset \mathbf{L}_{\mathbf{2}}^{\mathbf{1}}$, then since $\mathbf{L}_{\mathbf{2}}^{\mathbf{1}} \subset \mathbf{U M}$ but $D$ is not a union of monoids, $D \notin \mathbf{V}$ so, by the theorem, $\mathbf{V} \subseteq \mathbf{S M}$. In that event, $\mathbf{V} \cap \mathbf{M}=\mathbf{M S L}$, so by Theorem 3.5, $\mathbf{V}=\mathbf{S L} \vee \mathbf{M S L}$ and $\mathbf{L}_{2}^{1}$ covers $\mathbf{S L} \vee \mathbf{M S L}$.

COROLLARY 3.22 $\mathrm{D} \cap \mathrm{L}_{2}^{1}=\mathrm{SL}$.

Proof. This is clear from Propositions 3.18 and 3.21.

Note that, by Proposition 3.15, $\mathbf{V}\left(B_{01}^{+}\right)=\mathbf{L}_{\mathbf{2}}^{\mathbf{1}} \vee \mathbf{M C}$. 


\section{Appendix}

The material in this paper is self-contained, in that only the defining identities are needed. Gould [11] was the first to make explicit the identification of the varietal definitions of [left] restriction semigroups with the 'traditional' definitions of weakly [left] $E$-ample semigroups, and it was her paper that motivated the author to investigate the lattices of varieties. The later paper by Hollings [13] surveyed 'the historical development of the study of left restriction semigroups, from the 'weakly left E-ample' perspective', taking as the definition of left restriction semigroups, however, the semigroups of partial mappings of a given set that are closed under taking the identity maps on their domains.

Together, those two papers demonstrate the equivalence of these three approaches to the topic. They also provide a broad overview of the development of the various historical strands of development of the topic, including some not touched upon here, to which we refer the reader.

Here we briefly summarize these equivalences, so as to place our paper in context. Naturally, the reader is referred to [11] and [13] for a fuller exposition.

Let $S$ be a semigroup and let $E$ be a nonempty 'distinguished' subsemilattice of $E_{S}$. Define the relation $\widetilde{\mathcal{R}}_{E}$ on $S$ by a $\widetilde{\mathcal{R}}_{E} b$ if, for all $e \in E$, ea $=a$ if and only if $e b=b$. Each $\widetilde{\mathcal{R}}_{E}$-class of $S$ contains at most one member of $E$. Call $S$ weakly left $E$-ample if

(1) every element $a$ of $S$ is $\widetilde{\mathcal{R}}_{E}$-related to a (necessarily unique) member of $E$, which may be denoted $a^{+}$

(2) $\widetilde{\mathcal{R}}_{E}$ is a left congruence;

(3) for all $a \in S, e \in E$, ae $=(a e)^{+} a$.

Treating $S$ now as a unary semigroup $\left(S, \cdot{ }^{+}\right)$, and referring to the defining identities for left restriction semigroups in Section 1, notice that $E=\left\{x \in S: x^{+}=x\right\}$, so $\left(x^{+}\right)^{+}=x^{+}$ holds, the identities $x^{+} x=x$ and $x^{+} y^{+}=y^{+} x^{+}$are obvious, the identity $(x y)^{+}=\left(x y^{+}\right)^{+}$ (see Lemma 1.1) follows from (2), and the left ample identity $x y^{+}=(x y)^{+} x$ is an immediate consequence. Also as a result of that additional identity, $\left(x^{+} y\right)^{+}=\left(x^{+} y^{+}\right)^{+}=x^{+} y^{+}$.

Therefore every weakly left $E$-ample semigroup, regarded as a unary semigroup, satisfies the identities that we have used to define left restriction semigroups, and $E$ is its set of projections. Conversely, given any left restriction semigroup $\left(S, \cdot{ }^{+}\right)$and putting $E=P_{S}$, then $a \widetilde{\mathcal{R}}_{E} b$ if and only if $a^{+}=b^{+}$, that is, $a \mathbb{R} b$ in our notation, from which it readily follows that $S$ is weakly left $E$-ample.

When regarded from the varietal point of view, the semilattice of 'distinguished idempotents' is now no longer 'distinguished': it is simply the semilattice of projections, subsidiary to the unary operation. Thus the subscript notation on the generalized Green's relations plays only the historical role of distinguishing these semigroups from the earlier classes considered in the next paragraph. That is why we have chosen to start afresh with the notation $\mathbb{R}$, etc. A further reason is that these relations do behave in many ways like the 'usual' Green's relations, as will be more clearly seen in the sequel [17], where 'partial egg-boxes' will play a central role.

The term weakly left ample is reserved for the special case that $E=E_{S}$. The term left ample refers to the case that $\widetilde{\mathcal{R}}_{E}=\mathcal{R}^{*}$, the 'potential' Green's relation given by $a \mathcal{R}^{*} b$ if $x a=y a$ if 
and only if $x b=y b$ for all $x, y \in S^{1}$. (Necessarily, $E=E_{S}$ [11].) The inverse semigroups, and their full subsemigroups, provide a ready source of left ample semigroups. (See also Result 4.1 below.)

From the universal algebraic point of view, the great advantage of working with left restriction semigroups is that they form a variety. The weakly left ample and the left ample semigroups form only quasi-varieties. At least in the author's view, they also exhibit the most natural generality, in that the reduced left restriction semigroups comprise all monoids, whereas in the case of weakly left ample and left ample semigroups, they yield instead the unipotent and the right cancellative monoids, respectively, (see, for example, [10, Proposition 2.5]).

The explicit correspondence between weakly left $E$-ample semigroups and semigroups of partial mappings, which goes back in its essence to Trokhimenko [23], may also be found in $[11,13]$. Denote by $\mathcal{P} \mathcal{T}_{X}$ the semigroup of partial mappings of a nonempty set $X$, under composition, and for $\alpha \in \mathcal{P} \mathcal{T}_{X}$, let $\alpha^{+}$be the identity map on the domain of $\alpha$. Within $\mathcal{P} \mathcal{T}_{X}$ lies the inverse semigroup $\mathcal{I}_{X}$ of partial one-one mappings of $X$, under the natural inverse.

RESULT 4.1 The unary semigroup $\left(\mathcal{P} \mathcal{T}_{X}, \circ,{ }^{+}\right)$is a weakly left E-ample semigroup, the semilattice of projections consisting of the identity mappings on subsets of $X$. Conversely, any weakly left E-ample semigroup is (unarily) isomorphic to a unary subsemigroup of such a semigroup. The representation is by one-one mappings if and only if the semigroup is left ample.

The two-sided connections are established similarly. However, there is apparently no twosided analogue of this last result.

\section{References}

[1] S. Burris and H.P Sankappanavar, A Course in Universal Algebra, Springer-Verlag, Berlin, 1981.

[2] J.R. Cockett and S. Lack, Restriction categories I: categories of partial maps, Theoretical Computer Science 270 (2002), 223-259.

[3] C. Cornock, Restriction Semigroups: Structure, Varieties and Presentations, Ph.D. Thesis, 2011.

[4] L. Descalço and N. Ruškuc, Subsemigroups of the bicyclic monoid, Internat. J. Algebra Comput. 15 (2005), 37-57.

[5] L. Descalço and N. Ruškuc, Generalized Green's equivalences on the subsemigroups of the bicyclic monoid, Comm. Algebra 38 (2010), 45974612.

[6] C.C. Edmunds, Varieties generated by semigroups of order four, Semigroup Forum 21 (1980), 67-81.

[7] J. Fountain, A class of right PP monoids, Quart. J. Math Oxford 28 (1977), 285-300. 
[8] J. Fountain, Adequate semigroups, Proc. Edinburgh Math. Soc. 22 (1979), 113-125.

[9] J. Fountain, Free right type A semigroups, Glasgow Math. J. 33 (1991), 135-148.

[10] J. Fountain, G. Gomes, V.A.R. Gould, The free ample monoid, Internat. J. Algebra Comput. 19 (2009), 527-554.

[11] V.A.R. Gould, Notes on restriction semigroups and related structures (unpublished notes, available at www-users.york.ac.uk/ varg1/restriction.pdf).

[12] V.A.R. Gould and C. Hollings, Restriction semigroups and inductive constellations, Comm. Algebra 38 (2010), 261287.

[13] Christopher Hollings, From right $P P$ monoids to restriction semigroups: a survey, European J. Pure Appl. Math. 2 (2009), 21-57.

[14] John M. Howie, Fundamentals of Semigroup Theory, London Math. Soc. Monographs, Clarendon, (Oxford) 1995.

[15] M. Jackson and T. Stokes, An invitation to $C$-semigroups, Semigroup Forum 62 (2001), 279-310.

[16] Peter R. Jones, Varieties of $P$-restriction semigroups, submitted.

[17] Peter R. Jones, The semigroups $B_{2}$ and $B_{0}$ are inherently nonfinitely based, as restriction semigroups, submitted.

[18] Edmond W.H. Lee, Identity bases for some non-exact varieties, Semigroup Forum 68 (2004), 445-457.

[19] Yanfeng Luo and Wenting Zhang, On the variety generated by all semigroups of order three, J. Algebra 334 (2011), 1-30.

[20] S.O. Makanjuola and A. Umar, On a certain subsemigroup of the bicyclic semigroup, Comm. Algebra 25 (1997), 509-519.

[21] W.D. Munn, Free inverse semigroups, Proc. London Math. Soc. (3) 29 (1974), 385-404.

[22] M. Petrich, 'Inverse Semigroups', Wiley (New York), 1984.

[23] V.S. Trokhimenko, Menger's function systems, Izv. Vyssh. Uchebn, Zaved. Mat. 11(138) (1973), 71-78 (in Russian).

Department of Mathematics, Statistics and Computer Science

Marquette University

Milwaukee, WI 53201, USA

peter.jones@mu.edu 